\title{
House dust mite-induced seasonal and perennial allergic conjunctivitis in southwest Chinese children: case-control study
}

\section{Xu Gao}

Bi Shan Hospital of Chongqing

\section{Xiaojiao Tang}

Chongqing Medical University Affiliated Children's Hospital

Qin Xiang ( $D$ 164233023@qq.com)

Chongqing Medical University https://orcid.org/0000-0002-1992-0590

\section{Research article}

Keywords: Allergic conjunctivitis, seasonal allergic conjunctivitis, perennial allergic conjunctivitis, house dust mites, allergic diseases, skin prick test

Posted Date: December 10th, 2019

DOI: https://doi.org/10.21203/rs.2.18507/v1

License: (c) (i) This work is licensed under a Creative Commons Attribution 4.0 International License. Read Full License 


\section{Abstract}

Background: Seasonal (SAC) and perennial allergic conjunctivitis (PAC) are the most common allergic conjunctivitis. House dust mites are the most common sensitizing agents for SAC and PAC. This study aimed to explore the risk factors of allergic conjunctivitis in children.

Methods: The study recruited 176 children suffering from SAC or PAC and 131 normal control subjects in southwest of China. A questionnaire was provided to all the subjects. Several eye exams were also performed. All the subjects received the skin prick test (SPT). Then we analyzed the scores of the symptom, sign and the corneal fluorescein staining, the questionnaire data and the SPT results.

Results: The percent of the ever breast-fed in the case group was lower than that in control group $(P<0.05)$. The rate of parental allergy history in the case group was higher than that of the control group $(P<0.01)$. The scores of both ocular symptoms and signs had significant correlation to the clinical course $(P<0.05)$, but there was no relationship between these scores and the results of SPT $(P>0.05)$. The rate of parental allergy history in the case group was higher than that in the control group $(P<0.01$ both in father and mother). Compared with control group, the case group was more likely related to other systemic allergic disease $(P<0.01)$.

Conclusion: Allergic conjunctivitis might coexist with a variety of allergic diseases. Breastfeeding can effectively reduce the incidence of allergic conjunctivitis, especially for those children whose parents had history of allergic diseases.

\section{Background}

Allergic conjunctivitis (AC) is a group of diseases stimulated by allergens to conjunctiva, usually associated with type I (IgE-mediated hypersensitivity) and type IV hypersensitivity reactions (non-IgEmediated hypersensitivity). Although there are several classifications for ocular allergic disorders based on either clinical signs and symptoms or pathophysiology, the most commonly used classification are 2 acute disorders, including seasonal (SAC) and perennial allergic conjunctivitis (PAC), and 3 chronic diseases, including vernal keratoconjunctivitis (VKC), atopic keratoconjunctivitis (AKC) and giant papillary conjunctivitis (GPC) [1].

In China, we lack epidemiological investigation of allergic conjunctivitis, especially in children. The epidemiological investigations of allergic conjunctivitis in children are demanded badly. The majority cases of ocular allergy are SAC and PAC, whereas the severe styles such as AKC and VKC affect a smaller group of patients [2]. In America, PAC accounts for more than $90 \%$ patients with allergic conjunctivitis [3]. The prevalence of allergic conjunctivitis is increasing among Asian children [3]. In our country, PAC and SAC accounts for $74 \%$ patients with allergic conjunctivitis [4].

SAC and PAC are type I (IgE-mediated hypersensitivity) reactions [4]. Although SAC and PAC are mild, they affect the quality of life of a large number of children and adults [5]. PAC is considered to be a variant of 
SAC that persists throughout the year, $79 \%$ of patients who have PAC experience a seasonal exacerbation [6]. SAC and PAC are always accompanied by symptoms of allergic rhinitis [7].

There are differences in patterns of sensitizations in patients from different geographical areas. House dust mites are the most prevalent allergens in patients with asthma and/or rhinitis in China [8]. It is widely distributed, particularly in the southwest of China because of humid climate. House dust mites include dematophagoides farinae and dermatophagoides pteronyssinus [9]. They are the most common sensitizing agents for SAC and PAC $[10,11]$.

Although conjunctival provocation tests are an established diagnostic procedure for allergic conjunctivitis, they are not usually used in clinical regarding the relative risks for children [12]. Skin prick test (SPT) has higher accuracy in the diagnosis compared with measuring serum-specific lgE in vitro [2].

SPT has been wildly used as an easily and reliable diagnostic tool for evaluation the allergic status of individuals [13]. SPT is used to detect IgE-mediated hypersensitivity with $70-95 \%$ specificity and $80-97 \%$ sensitivity [14].

Here, we applied a questionnaire to a cohort of subjects with allergic conjunctivitis. 176 children with SAC or PAC, all from southwest of China, were studied for more details to explore the risk factors of allergic conjunctivitis in children and to analyze the related factors affecting the severity of clinical symptoms, hope to provide more help for clinical diagnosis and treatment in the future.

\section{Methods}

The experiment consisted of a disease-specific questionnaire, ophthalmologic examination, and SPT.

\section{Patients}

The study included a total of 176 subjects suffering from SAC or PAC with a positive history and skin tests, and 131 normal control subjects in Children's Hospital of Chongqing Medical University (Chongqing, China) from July 2015 to July 2017. Informed consent was obtained from one or both parents. The diagnosis of ocular allergy was based on clinical history, signs and symptoms, with the support of positive SPT. They had not previously been treated with topical or systemic H1-receptor antagonists, topical cromolyn or glucocorticosteroids.

\section{The disease-specific questionnaire and ophthalmologic examination}

A questionnaire included the basic characteristics, history of breastfeeding parental history of allergic disease, systemic allergic disease and the onset of season was performed in all subjects. 
Several eye exams included primary ophthalmologic examination, slit lamp observation, corneal fluorescent staining, tear break time (BUT) were carried out by our well-trained ophthalmologists.

The symptom score and sign score measurements were adapted from Macy M. S. Wu et al. (Table 1) [5, 15]. The corneal fluorescein staining scores were modified according to the National Eye Institute grading scale (Table

Table 1. Criteria for scoring symptoms and signs of perennial allergic conjunctivitis.

\begin{tabular}{|c|c|c|c|c|}
\hline \multirow[b]{2}{*}{ Item } & \multicolumn{4}{|c|}{ Scores } \\
\hline & & 1 & 2 & 3 \\
\hline \multicolumn{5}{|l|}{ symptoms } \\
\hline Rubbing eyes & No & mild & moderate & severe \\
\hline Itching & No & occasional & frequent & constant \\
\hline Blink & No & occasional & frequent & constant \\
\hline Redness & No & mild & moderate & severe \\
\hline \multicolumn{5}{|l|}{ signs } \\
\hline Chemosis & No & $\begin{array}{l}\text { one } \\
\text { quadrant }\end{array}$ & two to three quadrants & four quadrants \\
\hline $\begin{array}{l}\text { Tarsal conjunctival papillary } \\
\text { hypertrophy }\end{array}$ & No & mild & $\begin{array}{l}\text { moderate, }<1 / 3 \text { palpebral } \\
\text { conjunctival area }\end{array}$ & $\begin{array}{l}\text { severe, appear visualization of the deep } \\
\text { tarsal vessels }\end{array}$ \\
\hline Bulbar conjunctival hyperemia & No & mild & moderate & severe \\
\hline Keratitis & No & $\begin{array}{l}\text { one } \\
\text { quadrant }\end{array}$ & two quadrants & three or four quadrants \\
\hline Discoloration & No & mild & moderate & severe \\
\hline Mucus secretions & No & $\begin{array}{l}\text { small } \\
\text { amount }\end{array}$ & moderate amount & eyelid was glued in morning \\
\hline Limbal hypertrophy & No & $\begin{array}{l}\text { one } \\
\text { quadrant }\end{array}$ & two to three quadrants & more than three quadrants \\
\hline
\end{tabular}

\section{Skin prick test (SPT)}

The SPT tests were performed by the same nurse in a standardized manner. The patient's antihistaminic medication, if any, was stopped at least three days before the test. A drop of the allergen solution at standard activity and concentration was placed on the skin and pricked by a lancet. The lancet used for pricking the skin was a metallic lancet with 26 gauge. A prick made with a clean lancet was used as the negative control. The skin reaction was graded 20 minutes later. The skin edema and erythema that developed were graded from zero to four degrees by comparing the size with positive (histamine hydrochloride $1 \mathrm{mg} / \mathrm{ml}$ ) and negative (antigen diluting solution) controls [16]. Grade 1 is $25 \%$ of area of histamine-induced wheal, Grade 2 is $50 \%$ of this area, Grade 3 is $100 \%$ of this area, Grade 4 is $200 \%$ of it. Only grade 2, 3, and 4 were considered as positive skin reactions [17]. 


\section{Statistics}

The intergroup indicators of different groups (cases, controls) were described and compared. The quantitative data were described as mean \pm standard deviation (SD) and compared by the t-test. The rank data were described by median (quartile spacing), and the rank sum test of Wilcoxon or kruskal-wallis was used for inter-group comparison. Qualitative data were described by frequency (percentage), and chisquare test was used to compare the groups. All hypotheses were tested on both sides of the P value. A P-value 0.05 was set to be statistically significant. The confidence interval $(\mathrm{Cl})$ confidence interval was 95\%. SPSS software (version 21.0) was used for all statistical calculations (SPSS,Inc.,Chicago, IL, USA).

\section{Results}

\section{Clinical features}

A total of 307 subjects received questionnaires aged from 4 to 8 years. 176 subjects of those were allergic conjunctivitis, and 131 subjects were normal control. The mean age at first examination was 5.99 \pm 5.98 years. There was no significant difference in age between the two groups. The morbidity of allergic conjunctivitis was higher in male than in female of children $(P<0.01)$ (Table 2$)$.

\section{The onset of season}

Subjects were also asked about which season they experienced allergic conjunctivitis. A larger percentage of them were attacked in the spring (from April to May) and autumn (from September to October), almost abated during the winter. 18.18\% patients occurred PAC throughout the whole year without remission intermission (Fig. 1).

\section{Bedtime routines}

Child bedtime routines have been questioned through the survey. There was no difference between the two groups $(p>0.05)$, the results were showed in sTable 1 (supplementary Table 1 ).

\section{History of breastfeeding}

The parents were asked about if the children were got exclusive breast feeding and the duration of it. The relationship between breast-feeding and allergic conjunctivitis outcomes were investigated, the result showed that a small number of children in the case group were ever breast-fed and the percentage was far less than the control group $(19.85 \%$ VS 63.64\%) $(P<0.01)$. Among these who ever received breast-fed, the mean duration of exclusive breast-feeding in case group were much shorter than control group $(\mathrm{P}<$ 0.05) (Table 3). We explored the correlation between duration of exclusive breast-feeding and the age of 
onset, but no positive result was found. We also explored the correlation between the duration of exclusive breast-feeding and the degree of allergic reaction to different house dust allergens in relation to SPT, but no positive result was found (data were not shown).

Table 2

The clinical features of all the objects.

\begin{tabular}{|lllll|}
\hline & - & Control group & Case group & P value \\
\hline Age & Mean \pm SD & $5.92 \pm 2.51$ & $6.21 \pm 2.74$ & 0.336 \\
\hline & M (Q1 - Q3) & $6.00(4.08-8.17)$ & $5.83(4.00-7.42)$ & \\
\hline & Min - Max & $1.00-13.08$ & $1.00-15.00$ & \\
Sex & Male & $70(53.44 \%)$ & $46(26.14 \%)$ & $<0.01$ \\
\hline & Female & $61(46.56 \%)$ & $130(73.86 \%)$ & \\
\hline
\end{tabular}

Table 3

Relationship between breast-feeding and allergic conjunctivitis outcomes.

\begin{tabular}{|lllll|}
\hline & & Control group & Case group & P value \\
\hline Ever breast-fed & No & $26(19.85 \%)$ & $112(63.64 \%)$ & $<0.01$ \\
\hline & Yes & $105(80.15 \%)$ & $64(36.36 \%)$ & \\
\hline $\begin{array}{l}\text { Duration of exclusive } \\
\text { breast-feeding (months) }\end{array}$ & Means & $9.22 \pm 4.74$ & $7.82 \pm 3.47$ & 0.014 \\
\hline
\end{tabular}

\section{Parental history of allergic disease}

Parents were asked the history of allergic diseases such as allergic rhinitis, eczema, and urticaria. The rate of parental allergy history in the case group was higher than that in the control group $(P<0.01$ both in father and mother) (Table 4). The proportion of allergic rhinitis was the largest, which was $18.69 \%(n=$ $20)$ in father and $24.18 \%(n=22)$ in mother. 8 children in case group with both parents had the history of allergic disease which was allergic rhinitis yet. 
Table 4

The relationship between parental allergic history and the onset of perennial allergic conjunctivitis

\begin{tabular}{|lllll|}
\hline & & Control group & Case group & P value \\
\hline Father & 0 & $127(96.95 \%)$ & $107(60.80 \%)$ & $<0.01$ \\
\hline & 1 & $4(3.05 \%)$ & $69(39.20 \%)$ & \\
\hline mother & 0 & $129(98.47 \%)$ & $97(55.11 \%)$ & $<0.01$ \\
\hline & 1 & $2(1.53 \%)$ & $79(44.89 \%)$ & \\
\hline Both & 0 & $131(100 \%)$ & $173(98.30 \%)$ & 0.26 \\
\hline & 1 & $0(0 \%)$ & $3(1.70 \%)$ & \\
\hline 0
\end{tabular}

\section{Systemic allergic disease and allergic conjunctivitis}

We studied the relationship between systemic allergic diseases and allergic conjunctivitis in children. In the case group, allergic rhinitis was the most closely related to allergic conjunctivitis which was $85.80 \%$, followed by eczema, which was $76.14 \%$. The third was asthma, with $65.34 \%$. Besides, the atopic dermatitis and urticaria papulosa were also associated with allergic conjunctivitis. Compared with control group, these findings were all had significant differences (all $\mathrm{P}<0.01$ ). Surprisingly, we found some patients had adenoidal hypertrophy, although the proportion was not high which was $6.25 \%$, there was statistic difference between case group and control group $(P<0.05)($ Table 5$)$. 
Table 5

The subjects with systemic allergic diseases in two groups.

\begin{tabular}{|c|c|c|c|c|}
\hline & & Control group & Case group & $P$ value \\
\hline \multirow[t]{2}{*}{ Allergic rhinitis } & 0 & $126(96.18 \%)$ & $25(14.20 \%)$ & $<0.01$ \\
\hline & 1 & $5(3.82 \%)$ & $151(85.80 \%)$ & \\
\hline \multirow[t]{2}{*}{ Asthma } & 0 & $122(93.13 \%)$ & $61(34.66 \%)$ & $<0.01$ \\
\hline & 1 & $9(6.87 \%)$ & $115(65.34 \%)$ & \\
\hline \multirow[t]{2}{*}{ Atopic dermatitis } & 0 & $131(100.00 \%)$ & $141(80.11 \%)$ & $<0.01$ \\
\hline & 1 & $0(0.00 \%)$ & $35(19.89 \%)$ & \\
\hline \multirow[t]{2}{*}{ Urticaria papulosa } & 0 & $122(92.86 \%)$ & $97(55.56 \%)$ & $<0.01$ \\
\hline & 1 & $9(7.14 \%)$ & $79(44.44 \%)$ & \\
\hline \multirow[t]{2}{*}{ Eczema } & 0 & $116(88.55 \%)$ & $42(23.86 \%)$ & $<0.01$ \\
\hline & 1 & $15(11.45 \%)$ & $134(76.14 \%)$ & \\
\hline \multirow[t]{2}{*}{ Adenoidal hypertrophy } & 0 & $129(98.47 \%)$ & $165(93.75 \%)$ & 0.048 \\
\hline & 1 & $2(1.53 \%)$ & $11(6.25 \%)$ & \\
\hline \multicolumn{5}{|c|}{0 means without this disease. } \\
\hline \multicolumn{5}{|c|}{1 means with this disease. } \\
\hline
\end{tabular}

\section{Eyes symptoms}

The symptoms of PAC in children mainly included rubbing eyes, itching, blinking and redness. Almost half of children in case group sufferd from rubbing eyes, itching and blinking, those three symptoms were the most common in PAC children. The redness also could not be ignored which though was $26.7 \%$, as it was hard to distinguish from conjunctivitis (Fig. 2).

\section{Ocular signs}

The top three clinically signs of allergic conjunctivitis were chemosis, tarsal conjunctival papillary hypertrophy, and bulbar conjunctival hyperemia, but they were not more than $50 \%$ and lack of specificity. Interestingly, discoloration was specific and accounted for $21 \%(n=37)$, slightly lower than the top three signs but that was a pretty big proportion. Limbal hypertrophy was also specific and the proportion was $13.6 \%(n=24)$, which was lower than discoloration but it still always happened. The keratitis and mucus secretions were uncommon, with proportion of $8.0 \%$ and $9.7 \%$ respectively, as previously reported that 
involvement of corneal was rare [6] (Fig. 3). Moreover, only 2.84\% $(n=5)$ patients had scales and scurf of eyelid skin, and $2.27 \%(n=4)$ cases had meibomian gland obstruction (data were not shown).

\section{The relationship between the ocular symptom/sign scores and the duration of the disease}

The mean clinical course in case group was $3.09 \pm 2.92$ months. We found significant positive correlations between scores of ocular symptom/sign and the duration of the disease (Table 6).

Table 6

The correlations between scores of ocular symptom/sign and the duration of the disease.

\begin{tabular}{|llll|}
\hline & & Clinical course (months) & Mean \pm SD \\
\hline Sign scores & rs & 0.81 & $4.91 \pm 3.73$ \\
\cline { 2 - 4 } & P value & $<0.01$ & \\
Symptom scores & rs & 0.85 & $7.49 \pm 4.66$ \\
\cline { 2 - 4 } & P value & $<0.01$ & \\
\cline { 2 - 4 } & &
\end{tabular}

\section{The relationship between the ocular symptom/sign scores and results in SPT of two dust mite allergens}

The results of SPT in the control group were all negative. In case group, the results of skin reaction to dermatophagoides pteronyssinus were highly matched with the results of dermatophagoides farinae, the goodness of fit was up to $81.25 \%(n=143)$. Grade 3 was the mainly results of SPT for both dust mite allergens (Table 7). 
Table 7

Grading of the skin prick testing results in case group.

\begin{tabular}{|c|c|c|c|}
\hline & Grading & $\mathbf{N}$ & $\%$ \\
\hline \multirow{4}{*}{$\begin{array}{l}\text { Dermatophagoides } \\
\text { pteronyssinus }\end{array}$} & Grade 1 & 34 & $19.32 \%$ \\
\hline & Grade 2 & 38 & $21.59 \%$ \\
\hline & Grade 3 & 70 & $39.77 \%$ \\
\hline & Grade 4 & 34 & $19.32 \%$ \\
\hline \multirow[t]{4}{*}{ Dermatophagoides farinae } & Grade 1 & 42 & $23.86 \%$ \\
\hline & Grade 2 & 24 & $13.64 \%$ \\
\hline & Grade 3 & 76 & $43.18 \%$ \\
\hline & Grade 4 & 34 & $19.32 \%$ \\
\hline
\end{tabular}

We investigated the correlation between the ocular symptom/sign scores and the grading of SPT results response to these two dust mite allergens, but found no correlation. The results were shown in the supplementary Table 2 (sTable 2).

\section{Discussion}

This study included 307 subjects aged from 4 to 8 years. 176 subjects of those were allergic conjunctivitis, and 131 subjects were normal control.

Allergic conjunctivitis always happened in spring and autumn,seldom in winter. A larger percentage of patients were PAC (18.18\%), who happened at all seasons, throughout the whole year without remission.

The results of skin reaction to dermatophagoides pteronyssinus and dermatophagoides farinae were highly matched, which indicated these two dust mites may have the same allergic ingredients. Although there was no correlation was found between the ocular symptom/sign scores and the grades in SPT response to dust mite allergens, grade 3 was the most common. And we found there was a significantly positive correlations between scores of ocular symptom/sign and duration of the disease. And we found that the longer duration the disease lasted, the higher scores patients get (whether the symptoms or signs). It meant that lasting and allergic reaction to dust mite allergens, moderate or severer, may be responsible for the ocular symptoms and signs. This may explain that in our clinical, we found the ocular symptoms and signs were more severe in the children who were under desensitization treatment of dust mite allergens. 
Psychiatric factors may cause eyes blinking [18]. Eye blinking may have a transient tic disorder. When the course lasted long, it may be confused with tic, which could have some influence on the somatic symptoms and the patient's attention [19]. Psychiatric consultation may be necessary for children with long-term and frequent eye blinking. Incontrollable rubbing eyes would make the situation worse. So, it was important to provide proper intervention to alleviate their symptoms and signs.

In this questionnaire, the results showed that when in children, the morbidity of allergic conjunctivitis was higher in male than female. Generally, female would pay more attention to hygiene than male, this difference was especially obvious between boys and girls. We speculated that hygienic conditions maybe the key to the incidence of allergic conjunctivitis.

The results showed that the number of children in the case group who ever had exclusive breast-fed was far less than the control group. The mean duration of exclusive breast-feeding in case group was also shorter than the duration in control group. So, we suspected that exclusive breast-feeding maybe a protective factor for allergic conjunctivitis. Breast-feeding 4 months or more could reduce the risk of eczema and onset of the allergy marched to age 4, which was reported by Kull et al [20]. So we speculated that breast-feeding may play an important part in the onset of the allergic diseases and the duration of allergic reaction.

There was no correlation between the duration of exclusive breast-feeding and the age of onset or the degree of allergic reaction performed in SPT.

In addition, we found parental allergy history in the case group was significantly higher than that in control group, allergic rhinitis was the most common one, which meant parental allergy history especially with allergic rhinitis was a risk factor for their children to get allergic conjunctivitis.

Therefore, breast-feeding should be recommended as one possible way to reduce the risk of onset of allergic conjunctivitis, prolonged breast-feeding was particularly recommended for these infants with parental allergy history. These children with parental allergy history deserved special attention in the clinical treatment, especially during the inquiry.

Systemic allergic diseases were closely related with allergic conjunctivitis. According to our study, allergic rhinitis was the most common, followed by eczema, asthma and urticaria papulosa. So, inquiring of the systemic allergic history was necessary in clinic, especially for these children without typical symptoms and signs or too young to explain himself, this would help us to diagnose and give proper treatment.

Interestingly, children with the adenoidal hypertrophy (ATH) were more common in case group, which means allergic conjunctivitis may be a risk factor for ATH, this result was consistent with Modrzynski Metal [21-23]. Seasonal and perennial allergic conjunctivitis were considered to be associated with type I hypersensitivity reactions [24]. The conjunctiva located in the upper extremity of the respiratory system, the nasolacrimal duct was a drainage system in to the nose [25]. Allergens and allergic mediators would drain to the nose by this pathway, generating nasal symptoms. The conjunctiva and the nose made up an 
entire system $[1,2,5-8]$. Therefore, the coexistence of allergic rhinitis and conjunctivitis happened repeatedly.

Xiaowen Zhang et al found the rate of slgE presenting in adenoids or tonsils was significantly higher than that in the serum of childhood ATH, which suggested a role of local atopy [26, 27]. Allergy control may play some role in reducing the rate of adenotonsillectomy in children suffering from allergic reactions caused by ATH [28]. We assumed that in these children suffering from PAC combined with ATH, effectively controlment of PAC could alleviate the symptoms of ATH and reduce the rate of adenotonsillectomy. And children with ATH should have an ophthalmic exam to determine whether suffering from allergic conjunctivitis, in order to give proper synchronous treatments.

The symptoms of SAC and PAC in children were typical mainly including rubbing eyes, itching, blinking and redness. Nearly half of children had rubbing eyes, itching, and blinking, which were the top three symptoms. The top three clinical signs of allergic conjunctivitis were chemosis, tarsal conjunctival papillary hypertrophy, and bulbar conjunctival hyperemia, but they were not specific. Discoloration, limbal hypertrophy, mucus secretions and keratitis were the characteristic signs. Discoloration and limbal hypertrophy were always happened, the circumference of it could become thickened and opaque. The keratitis and mucus secretions were rare in our findings and often happened when rubbing eyes were uncontrollable [6]. The ocular surface inflammation usually was driven by mast cell which led to rubbing eyes, itching, blinking and redness in the acute phase [29]. So, PAC were traditionally treated with combination anti-histamine mast cell stabilizers. But in severe subjects, these drugs were not effective. A late-phase response developed by eosinophilia and neutrophilia should be considered, which may lead to remodel the ocular surface tissues manifesting discoloration and limbal hypertrophy.

Lately, in clinical, milder cases can be treated with short-term topical ophthalmic therapy such as decongestant/antihistamine combination, mast cell stabilizer, or multi-action agent. Moderate to severe cases may require longer use of the above agents or the addition of an oral antihistamine. Refractory cases may need the use of topical ophthalmic corticosteroids and/or immunotherapy. Corticosteroids had been proven effective, but the potential side effects including increased intraocular pressure, cataracts and corneal melts would restrict the duration of corticosteroid usage. After stopping the corticosteroids, the disease would be back and forth. Even though nasal corticosteroids were not a firstline treatment for allergic conjunctivitis, in patients with rhinoconjunctivitis, nasal mometasone furoate and fluticasone furoate had been shown to relieve the associated conjunctival symptoms. Although intranasal corticosteroids could improve ocular symptoms, the involved mechanism(s), probably related to reduction of nose-ocular reflex, still was unknown.

Inventing more effective medicine to treat seasonal and perennial allergic conjunctivitis were extremely urgent. Better understanding of the clinical parameters of the syndromes induced by allergic conjunctivitis and the cell and molecular basis of this disease was important to explore both safe and effective treatment. 
Children were different from adults. As more we learned about the allergic conjunctivitis in children, it may reduce the misdiagnose and misuse of antibiotics.

\section{Conclusion}

Allergic conjunctivitis was not only a local disease that affected the ocular surface, but also might coexist with a variety of allergic diseases. Therefore, it should pay more attention in the clinical practice to the children's systemic symptoms and the family histories in order to provide a good suggestion of treatment. Breastfeeding can effectively reduce the incidence of allergic conjunctivitis, especially for those children whose parents had history of allergic diseases, prolonged breast-feeding was particularly recommended.

\section{Abbreviations}

AC

Allergic Conjunctivitis

AKC

Atopic Keratoconjunctivitis

ATH

Adenoidal Hypertrophy

BUT

Tear Break Time

GPC

Giant Papillary Conjunctivitis

PAC

Perennial Allergic Conjunctivitis

SAC

Seasonal Allergic Conjunctivitis

SPT

Skin Prick Test

VKC

Vernal Keratoconjunctivitis

\section{Declarations}

The study followed the Tenets of the Declaration of Helsinki and was approved by the Ethics Committee of the Children's Hospital of Chongqing Medical University, Chongqing, China (Permit No.001/2013). Written informed consent was obtained from the parents of each subject. The study was registered in the Chinese Clinical Trial Registry (Registration number ChiCTR-OCC-14004140).

Consent for publication: Not applicable. 
Availability of data and material: The data that support the findings of this study are available from the Medical Records Room of Children's Hospital of Chongqing Medical University, but there were restrictions applied to the availability of these data, which were used under license for this current study, and so are not publicly available. Data are however available from the authors upon reasonable request and with permission of the Medical Records Room of Children's Hospital of Chongqing Medical University.

Competing interests: The authors declare that they have no competing interests

Funding: The study and the writing of the manuscript were supported by Chongqing Municipal Health Bureau (ZY201702067). The funding body no financial relations with this research.

Authors' contributions: XG was a major contributor in writing the manuscript, doing the statistical analysis and editing tables. QX revised the manuscript and helped perform the analysis with constructive discussions. XG, XT and QX completed the acquisition of data, analysis and interpretation of data together. QX made substantial contributions to conception and revised the manuscript, given final approval of the version to be published. All authors read and approved the final manuscript. Each author had participated sufficiently in the work to take public responsibility for appropriate portions of the content, agreed to be accountable for all aspects of the work in ensuring that questions related to the accuracy or integrity of any part of the work are appropriately investigated and resolved.

Acknowledgements: The authors thank all patients and their parents enrolled in the present study. We want to thank Xiurong Chen and Chunmei Yuan for recording patients' information in the first place.

\section{References}

1. Leonardi A, Bogacka E, Fauquert JL, Kowalski ML, Groblewska A, Jedrzejczak-Czechowicz M, Doan S, Marmouz F, Demoly P, Delgado L: Ocular allergy: recognizing and diagnosing hypersensitivity disorders of the ocular surface. Allergy 2012, 67(11):1327-1337.

2. Yanni JM, Barney NP: CHAPTER 11 - Ocular Allergy: Clinical, Therapeutic and Drug Discovery Considerations. Ocular Therapeutics 2008:239-274.

3. Fok AO, Wong GW: What have we learnt from ISAAC phase III in the Asia-Pacific rim? Current Opinion in Allergy \& Clinical Immunology 2009, 9(2):116-122.

4. Johansson SG, Hourihane JO, Bousquet J, Bruijnzeelkoomen C, Dreborg S, Haahtela T, Kowalski ML, Mygind N, Ring J, Van CP: A revised nomenclature for allergy. An EAACl position statement from the EAACI nomenclature task force. Allergy 2001, 56(9):813-824.

5. Thong YH: Allergic conjunctivitis in Asia. Asia Pacific Allergy 2017, 7(2):57-64.

6. Dart JK, Buckley RJ, Monnickendan M, Prasad J: Perennial allergic conjunctivitis: definition, clinical characteristics and prevalence. A comparison with seasonal allergic conjunctivitis. Transactions of the Ophthalmological Societies of the United Kingdom 1986, 105 ( pt 5)(5):513-520. 
7. Tong, Qiao, Yizhen, Zhinan, Wang: Pediatric allergic conjunctivitis and allergic rhinitis. The Journal of Biomedical Research 2008, 22(3):183-187.

8. Li J, Sun B, Huang Y, Lin X, Zhao D, Tan G, Wu J, Zhao H, Cao L, Zhong N: A multicentre study assessing the prevalence of sensitizations in patients with asthma and/or rhinitis in China. Allergy 2010, 64(7):1083-1092.

9. Fujisaki T, Hyo Y, Hamamoto M, Saika T, Harada T: Present Conditions of Patients with Nasal Allergies at the Department of Otolaryngology, Kawasaki Medical School-Rates of House Dust Mite and Dematophagoides farinae Allergen-specific IgE Antibody Positivity as Determined Using the CAPRAST System-. Practica oto-rhino-laryngologica Supp/2017, 149:152-157.

10. Neto HJ, Rosário NA, Westphal GL, Riedi CA, Santos HL: Allergic conjunctivitis in asthmatic children: as common as underreported. Annals of Allergy Asthma \& Immunology 2010, 105(5):399-400.

11. Riedi CA, Rosario NA: Prevalence of allergic conjunctivitis: a missed opportunity? Allergy 2010, 65(1):131-132.

12. Zdenek Pelikan MD: Seasonal and perennial allergic conjunctivitis: the possible role of nasal allergy. Australian \& New Zealand Journal of Ophthalmology 2010, 37(5):448-457.

13. Antunes J, Borrego L, Romeira A, Pinto P: Skin prick tests and allergy diagnosis. Allergologia Et Immunopathologia 2009, 37(3):155-164.

14. Caffarelli, Dascola, Povesi C, Ricci, Giampaolo, Dondi: Skin prick test to foods in childhood atopic eczema: pros and cons. Italian Journal of Pediatrics 2013, 39(1):48-48.

15. Wu MM, Yau GS, Lee JW, Wong AL, Tam VT, Yuen CY: Retrospective review on the use of topical cyclosporin a $\mathbf{0 . 0 5 \%}$ for paediatric allergic conjunctivitis in Hong Kong Chinese.

TheScientificWorldJournal 2014, 2014:396987.

16. Dibek ME, Reha CM: Skin prick test results of child patients diagnosed with bronchial asthma. Allergologia Et Immunopathologia 2007, 35(1):21-24.

17. Prasad R, Verma SK, Dua R, Kant S, Kushwaha RAS, Agarwal SP: A study of skin sensitivity to various allergens by skin prick test in patients of nasobronchial allergy. Lung India 2009, 26(3):7073.

18. Collins M, Seeto R, Campbell L, Ross M: Blinking and corneal sensitivity. Acta ophthalmologica 1989, 67(5):525-531.

19. Jung HY, Chung SJ, Hwang JM: Tic disorders in children with frequent eye blinking. Journal of AAPOS : the official publication of the American Association for Pediatric Ophthalmology and Strabismus 2004, 8(2):171-174.

20. Kull I, Böhme M, Wahlgren CF, Nordvall L, Pershagen G, Wickman M: Breast-feeding reduces the risk for childhood eczema. Journal of Allergy \& Clinical Immunology 2005, 116(3):657-661.

21. Karaca CT, Toros SZ, Noseri H, Kulekci S, Kalayck C, Oysu C, Klcoglu G, Egeli E: Role of allergy in children with adenotonsillar hypertrophy. The Journal of craniofacial surgery 2012, 23(6):e611-613. 
22. Modrzynski M, Zawisza $\mathrm{E}$, Mazurek $\mathrm{H}$ : [The analysis of incidence of adenoid hypertrophy in children hypersensitive to dust mites]. Otolaryngologia polska = The Polish otolaryngology 2006, 60(6):865871.

23. Sadeghi-Shabestari M, Jabbari Moghaddam $\mathrm{Y}$, Ghaharri $\mathrm{H}$ : Is there any correlation between allergy and adenotonsillar tissue hypertrophy? International journal of pediatric otorhinolaryngology 2011, 75(4):589-591.

24. Ono SJ, Abelson MB: Allergic conjunctivitis: update on pathophysiology and prospects for future treatment. The Journal of allergy and clinical immunology 2005, 115(1):118-122.

25. Bowling E, Russell GE: Treat the Itch Without a Hitch. Review of Optometry 2009(4).

26. Cho KS, Kim SH, Hong SL, Lee J, Mun SJ, Roh YE, Kim YM, Kim HY: Local Atopy in Childhood Adenotonsillar Hypertrophy. American journal of rhinology \& allergy 2018, 32(3):160-166.

27. Zhang $X$, Sun $B$, Li S, Jin H, Zhong N, Zeng G: Local atopy is more relevant than serum sigE in reflecting allergy in childhood adenotonsillar hypertrophy. Pediatric allergy and immunology : official publication of the European Society of Pediatric Allergy and Immunology 2013, 24(5):422-426.

28. Scadding G: Non-surgical treatment of adenoidal hypertrophy: the role of treating IgE-mediated inflammation. Pediatric allergy and immunology : official publication of the European Society of Pediatric Allergy and Immunology 2010, 21(8):1095-1106.

29. Ono SJ, Abelson MB: Allergic conjunctivitis: update on pathophysiology and prospects for future treatment. J Allergy Clin Immunol 2005, 115(1):118.

\section{Figures}




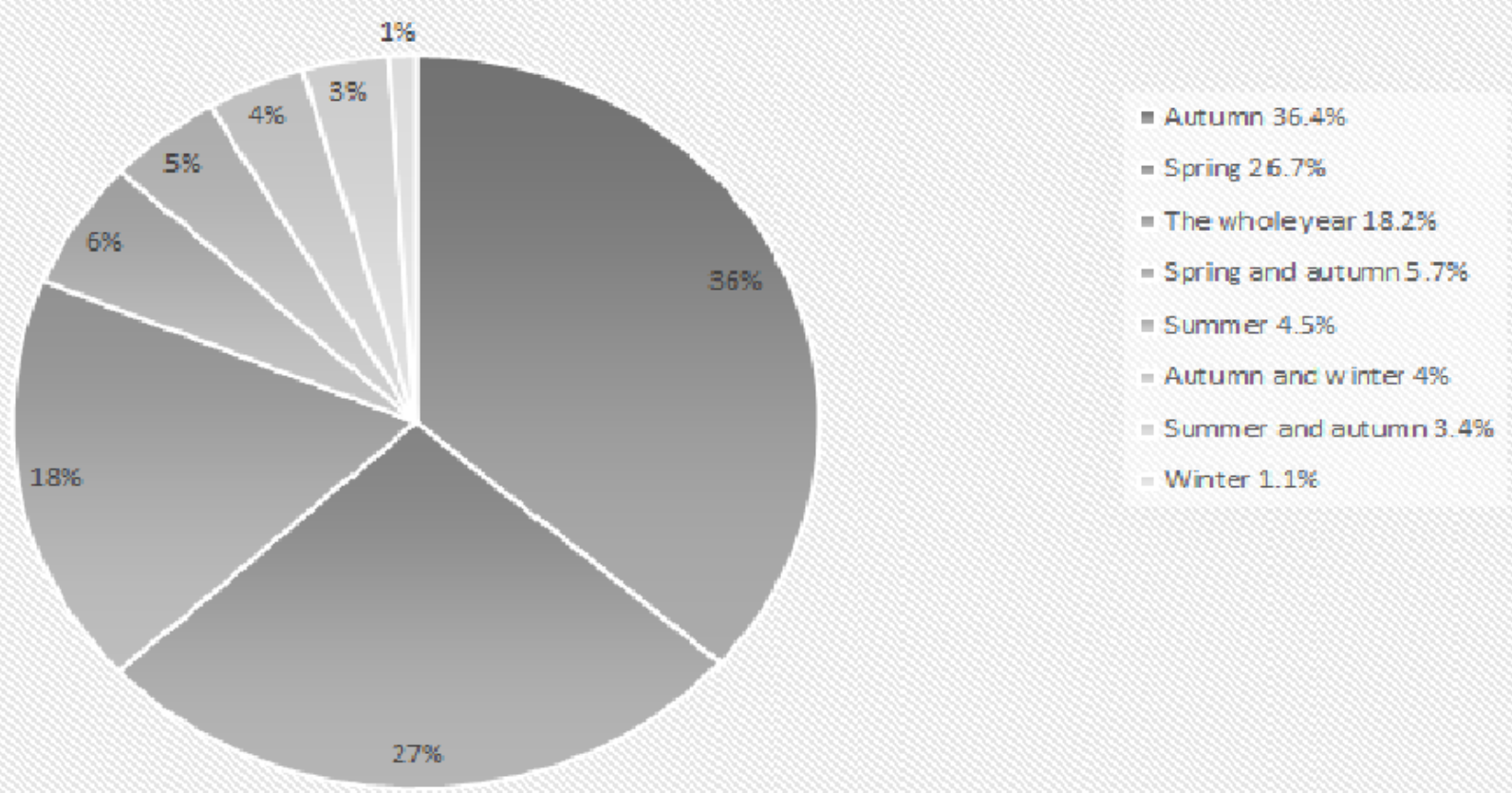

\section{Figure 1}

The percentages of symptoms in case group in the period of the year. $36.4 \%$ patients occurred in the autumn ( $n=64) ; 26.7 \%$ occurred in the spring $(n=47) ; 4.5 \%$ occurred in the summer $(n=8) ; 1.1 \%$ occurred in the winter $(n=2) ; 5.7 \%$ patient occurred in both the spring and autumn $(n=10) ; 3.4 \%$ occurred in both the summer and autumn $(n=6) ; 4.0 \%$ occurred in both the autumn and winter $(n=7) ; 18.2 \%$ patients occurred all the year around $(n=32)$. 